\title{
In-medium effects in central heavy ion collisions at intermediate energies
}

\author{
M. Henri, ${ }^{1},{ }^{*}$ O. Lopez,${ }^{1}$ D. Durand,${ }^{1}$ B. Borderie,${ }^{2}$ R. Bougault,${ }^{1}$ A. Chbihi,${ }^{3}$ \\ Q. Fable ${ }^{3}$ J.D. Frankland,${ }^{3}$ E. Galichet,${ }^{2,}{ }^{4}$ D. Gruyer ${ }^{1}$ J. Lemarié ${ }^{3}$ N. Le Neindre,${ }^{1}$ \\ I. Lombardo, ${ }^{5}$ M. Pârlog, ${ }^{1,6}$ J. Quicray, ${ }^{1}$ G. Verde $^{5}{ }^{5}$ E. Vient, ${ }^{1}$ and M. Vigilante ${ }^{7}$ \\ (INDRA collaboration) \\ ${ }^{1}$ Normandie Univ., ENSICAEN, UNICAEN, CNRS/IN2P3, LPC Caen, 14000 Caen, France \\ ${ }^{2}$ Université Paris-Saclay, CNRS/IN2P3, IJCLab, 91405 Orsay, France \\ ${ }^{3}$ Grand Accélérateur National d'Ions Lourds (GANIL), \\ CEA/DRF - CNRS/IN2P3, Bvd. Henri Becquerel, F-14076 Caen, France \\ ${ }^{4}$ Conservatoire National des Arts et Metiers, F-75141 Paris Cedex 03, France \\ ${ }^{5}$ INFN - Sezione Catania, via Santa Sofia 64, I-95123 Catania, Italy \\ 6 "Horia Hulubei" National Institute of Physics and Nuclear Engineering (IFIN-HH), RO-077125 Bucharest Magurele, Romania \\ ${ }^{7}$ Dipartimento di Fisica 'E. Pancini' and Sezione INFN, \\ Università di Napoli 'Federico II', I-80126 Napoli, Italy
}

(Dated: June 5, 2020)

\begin{abstract}
Energy dissipation and cluster production in central nuclear collisions in the Fermi energy range have been investigated using the large INDRA database. A study of the in-medium nucleon-nucleon cross section, $\sigma_{N N}^{*}$, has been performed by comparing the results of a model with the measured isotropy ratio of the kinetic energy distributions of the detected protons. This analysis has been done for a large variety of systems at various incident energies from 20 to $100 \mathrm{MeV} / \mathrm{nucleon}$ and confirms nicely previous results with enhanced statistics in a full Monte Carlo treatment. Most importantly, it is found that $\sigma_{N N}^{*}$ is drastically reduced as compared to the vacuum values, due partly to Pauli blocking but also to in-medium effects for which mean values and uncertainties are given. Concerning the cluster production, a coalescence model has been developed to study the parallel and perpendicular velocity distributions of the light clusters $(A \leq 4)$. For the $A_{C}=3\left({ }^{3} \mathrm{H}\right.$, ${ }^{3} \mathrm{He}$ ), it is found that the mean internal kinetic energy of the nucleons in the cluster is reduced as compared to the vacuum value. These two results shows that any comparison between experimental and theoretical predictions in heavy-ions induced reactions around and above Fermi energy must take into account these in-medium effects.
\end{abstract}

\section{INTRODUCTION}

Over the last decades, the Equation of State (EoS) of nuclear matter has been widely studied in nuclear reactions in order to put constraints on theoretical models. These latter aim to describe both nuclear properties at microscopic scale and astrophysical phenomena at macroscopic scale. In the first case, the EoS allows to understand some aspects of the collision via the underlying mechanisms like deep inelastic scattering, fusion reactions, collective motions to mention a few examples [1]. In the second one, the EoS allows to describe the heavy compact astrophysical objects, as for instance Core Collapse SuperNovae and the formation of neutron stars [2]. Transport properties of the nuclear medium, in particular the in-medium nucleon-nucleon cross section, $\sigma_{N N}^{*}$, and in-medium cluster production contribute to the determination of the EoS through properties of the residual strong interaction and constitute a fundamental ingredient for microscopic transport models [3].

In the Fermi energy range $(10-100 \mathrm{MeV} /$ nucleon $)$, the characteristics of the transport properties reflect the

\footnotetext{
* Present affiliation: Grand Accélérateur National d'Ions Lourds (GANIL), CEA/DRF - CNRS/IN2P3, Bvd. Henri Becquerel, F-14076 Caen, France
}

interplay between the mean field (via the so-called onebody dissipation) and the two-body dissipation essentially governed by $\sigma_{N N}^{*}[4,5]$. The transition between the two modes of dissipation is around the Fermi energy $E_{F} \approx 38 \mathrm{MeV} /$ nucleon. Well below $E_{F}$, the one-body dissipation as predominant mode has been investigated by the study of fusion cross-sections [6, 7,. Well above $E_{F}$, the dissipation mode is increasingly induced by $\mathrm{NN}$ collisions [8-10]. Thus, a transition around $E_{F}$ is observed, and corresponds to the crossover between onebody to two-body dissipation [8, 9. This phenomenon is related to in-medium effects on the transport properties of nucleons in nuclear matter. Thus, we wonder if these in-medium effects can also affect heavier nuclear species, here clusters produced in the hot and dense nuclear medium environment. Indeed, light clusters $\left(A_{C} \leq 4\right)$ could greatly impact the EoS and constitutes an important topic of study nowadays [11, 12. The modification of the clusters characteristics such as its binding energy in the nuclear medium due to the surrounding nuclear environment must be taken into account and investigated in order to better constrain the theoretical models [13 15.

In this work, we present two analyses focusing on the in-medium properties mentioned above. To this end, two similar models have been developed: the first for simulating elastic NN collisions above $E_{F}$ to study the energy 
dissipation, the second for simulating the nucleon coalescence process to address the question of in-medium cluster production. In section II] the experimental setup and the experimental dataset used for the analysis are presented. Section III focuses on the study of in-medium nucleon-nucleon collisions. The question of the cluster production in the nuclear medium using a coalescence model is presented in section IV. Finally, we summarize our results in section $\mathrm{V}$.

\section{EXPERIMENTAL SETUP AND CENTRAL EVENTS SELECTION}

\section{A. Experimental setup}

INDRA is a $4 \pi$ multidetector aiming at studying heavy ion collisions in the Fermi energy regime (10 $100 \mathrm{MeV} /$ nucleon). It is installed and in operation since 1993 at the Grand Accélerateur National d'Ions Lourds (GANIL) in Caen. A complete and detailed description of the detector and electronic setup can be found in [16, 17]; we present here only the main specifications. INDRA is composed of 336 detection modules, arranged in 17 rings with a cylindrical symmetry around the beam axis. The first ring $\left(2^{\circ}-3^{\circ}\right)$ used for the first two campaigns (symmetric systems: ${ }^{36} \mathrm{Ar}+\mathrm{KCl},{ }^{58} \mathrm{Ni}+{ }^{58} \mathrm{Ni} \&$ ${ }^{129} \mathrm{Xe}+{ }^{119} \mathrm{Sn}$ ) was composed of phoswich scintillators telescopes, replaced later by silicon-cesium iodide (SiCsI(Tl)). Rings 2 to $9\left(3^{\circ}-45^{\circ}\right)$ are composed of three stages telescopes. The first stage is an ionization chamber (IC) operated at low pressure in order to get very low detection and identification thresholds (1 MeV/nucleon). The second one is a silicon semiconductor ( $\mathrm{Si}$ ). Finally, the last stage is made of inorganic cesium iodide scintillator $\mathrm{CsI}(\mathrm{Tl})$ which plays an important role in the detection of the clusters [18 20]. The remaining rings 10 to 17 $\left(45^{\circ}-178^{\circ}\right)$ are made of IC-CsI $(\mathrm{Tl})$ telescopes. The experimental device provides thus a wide angular coverage of $90 \%$ of $4 \pi$, a charge (Z) identification from hydrogen up to uranium and an isotopic resolution ( $\mathrm{Z} \& \mathrm{~A}$ ) up to beryllium (before 2001) and oxygen (after 2001).

We have used in this work a large part of the symmetric systems studied with INDRA, covering incident energies from 15 to $100 \mathrm{MeV} /$ nucleon and total system mass from 72 to 394 uma (see table I).

\section{B. Central events selection}

In the following, we select a sample of central collisions, which are supposed to be the most dissipative and where the degree of equilibration should be the largest. We have used the method presented in [8]. This is based on the correlation between the isotropy ratio $R_{E}$ and the detected multiplicity of charged particles, $M_{c}$. A multiplicity cut, $M_{c u t}^{\text {low }}$, is used to select the most dissipative collisions. For multiplicities above a value $M_{\text {cut }}^{\text {low }}$ the

\begin{tabular}{ccc}
\hline \hline System & $E_{P}(\mathrm{MeV} /$ nucleon $)$ & Analysis \\
\hline${ }^{36} \mathrm{Ar}+\mathrm{KCl}$ & $32-74$ & $\bullet$ \\
${ }^{58} \mathrm{Ni}+{ }^{58} \mathrm{Ni}$ & $32-90$ & $\bullet \dagger$ \\
${ }^{129} \mathrm{Xe}+{ }^{\mathrm{nat}} \mathrm{Sn}$ & $12-50$ & $\bullet \dagger$ \\
${ }^{129} \mathrm{Xe}+{ }^{124} \mathrm{Sn}$ & $65-100$ & $\bullet \dagger$ \\
${ }^{136} \mathrm{Xe}+{ }^{124} \mathrm{Sn}$ & 32,45 & $\bullet$ \\
${ }^{136} \mathrm{Xe}+{ }^{112} \mathrm{Sn}$ & 32,45 & $\bullet$ \\
${ }^{124} \mathrm{Xe}+{ }^{124} \mathrm{Sn}$ & 32 & $\bullet$ \\
${ }^{124} \mathrm{Xe}+{ }^{112} \mathrm{Sn}$ & 32,45 & $\bullet$ \\
${ }^{197} \mathrm{Au}+{ }^{197} \mathrm{Au}$ & $40-100$ & $\bullet \dagger$ \\
\hline \hline
\end{tabular}

TABLE I. Table summarizing all the systems used in the analysis presented here. The $\bullet$ symbol indicates the systems used in the section III the $\uparrow$ the systems used in the section IV]

mean value of the isotropy ratio $R_{E}$ becomes constant. Assuming that isotropy and centrality are strongly correlated, we therefore deduce that $M_{c} \geq M_{\text {cut }}^{\text {low }}$ selects the most central collisions This corresponds to a fraction between 1 and $2 \%$ of the total measured cross section [8, 9]. Regarding the reduced impact parameter $\hat{b}=\frac{b}{b_{\max }}\left(b_{\max }\right.$ is the maximal impact parameter for a given system), this selection corresponds to a set of events where: $0<\hat{b} \leq 0.14$.

It is worthwhile to stress that, as already mention in [8], sources from quasifusion reactions can be selected using topology selectors 21] rather than the total charged product multiplicity.

\section{STUDY OF THE IN-MEDIUM NUCLEON-NUCLEON CROSS SECTION}

\section{A. Experimental results}

In this section, we study the kinematical properties of the protons (the neutrons are not detected with INDRA), as done in [9]. To this end, the isotropy ratio using the center-of-mass energy $R_{E}^{p}$, is defined as:

$$
R_{E}^{p}=\frac{1}{2} \cdot \frac{E_{\perp}^{c . m .}}{E_{\|}^{c . m} .}
$$

where $E_{\perp}^{c . m}$. and $E_{\|}^{c . m}$. are respectively the sum of the perpendicular and the parallel energies of all detected protons in the center of mass frame. Three regimes are possible; $R_{E}^{p}>1$ : the energy is preferentially distributed in the transverse plane of the reaction (the so-called squeeze-out); $R_{E}^{p}<1$ : the energy is preferentially distributed along the beam axis indicating a partial memory of the entrance channel; $R_{E}^{p}=1$, the energy is distributed isotropically corresponding to energy/momentum equilibration and hence thermalization.

Fig. 1 shows the evolution of the isotropy ratio $R_{E}^{p}$ for the 37 systems listed in table $\mathbb{1}$. The error bars correspond 
to the (quadratic) sum of statistical and systematics errors. These latter are estimated by varying the lower multiplicity cut value of two units $M_{\text {cut }}^{\text {low }} \pm 2$ for the central event selection. Doing so, we provide better error estimates than in 9] where only statistical errors were provided. Also, the statistics have been increased mostly by a factor of 10 by taking all the available amount of data. This is why the error bars in Fig. 1 are smaller than those of Fig. 1 in 9 . The red curve corresponds to the expected value of the entrance channel by considering two Fermi spheres separated by the relative velocity of the incoming nuclei. The blue horizontal line corresponds to an isotropic distribution of protons, assumed to correspond to full stopping.

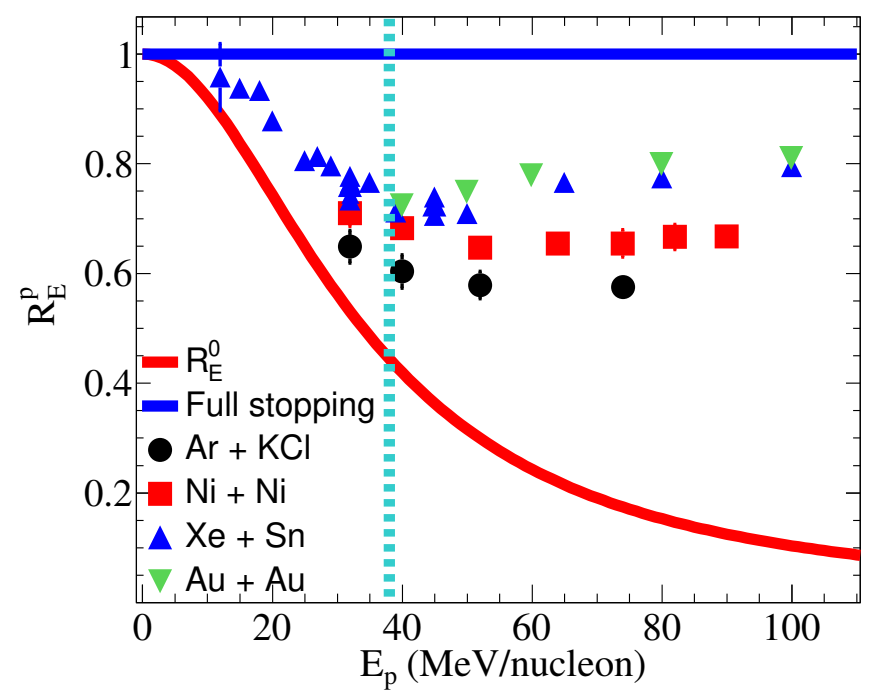

FIG. 1. (Color online) Evolution of the proton isotropy ratio $R_{E}^{p}$ as a function of the projectile energy in the laboratory $E_{P}$, for all studied systems. The red and blue lines correspond to the entrance channel and the full stopping (see text). The vertical dotted line is the Fermi energy.

As already mentioned in $[8$ and $[9$, the isotropy ratio scales according to the mass of the system. The lighter system $(\mathrm{Ar}+\mathrm{KCl})$ displays the smallest values while the heaviest one $(\mathrm{Au}+\mathrm{Au})$ is associated with the largest ones. All $R_{E}^{p}$ values are located between the two limiting regimes. This means that the dissipation of the entrance channel energy is not complete. A minimum is observed around $E_{F}=38 \mathrm{MeV} /$ nucleon and is presumably linked to the transition in the dissipation mode: one-body (mean field) below $E_{F}$ and two-body (nucleonnucleon collisions) above $E_{F}[8,9$. We here confirm globally the results obtained in $[9]$.

\section{B. Nucleon-nucleon collisions simulation}

In order to study the evolution of $R_{E}^{p}$ above $E_{F}$, we have built a simulation to study the evolution of the momentum and energy distributions of the nucleons mod- ified by in-medium nucleon-nucleon collisions. Initially, the momentum distribution of the nucleons inside the projectile $\left(Z_{P}, A_{P}\right)$ and the target $\left(Z_{T}, A_{T}\right)$ are sampled using a Fermi-Dirac distribution at zero temperature (sudden approximation). A full overlap of the projectile and the target in the position space is assumed due to the selection of central events and symmetric systems. Therefore, NN collisions are only performed in momentum space. Finally, we consider only elastic NN collisions since in the energy range considered, inelastic channels are marginally opened 22 .

NN collisions are considered by picking randomly a nucleon from the projectile and the target. While $n-n$ and $p-p$ collisions are isotropic [23], the free differential cross section regarding $n-p$ and $p-n$ collisions is taken from [24]:

$$
\begin{aligned}
\frac{d \sigma}{d \Omega}\left(E_{l a b}, \theta\right)= & \frac{17.42}{1+0.05\left(E_{l a b}^{0.7}-15.5\right)} \\
& \times \exp \left(b\left(\cos ^{2} \theta+\sin ^{2} \frac{\theta}{7}-1\right)\right)
\end{aligned}
$$

where $b=0.125\left(E_{l a b}^{0.54}-4.625\right)$ if $E_{l a b} \leq 100 \mathrm{MeV}$, and $b=0.065\left(36.65-E_{l a b}^{0.58}\right)$ if $E_{l a b}>100 \mathrm{MeV}$. Note that the values of $b$ given in the original article 24] are wrong and have been corrected in agreement with the authors 25]. The Pauli exclusion principle in the exit channel of the diffusion has to be taken into account by the so-called Pauli blocking factor. This is implemented here by rejecting the collisions for which either of the two scattered nucleon after the collision is located inside the initial momentum Fermi sphere, since such states are already occupied.

The mean number of collisions by nucleon $\frac{\left\langle N_{\text {coll }}\right\rangle}{A}$ for a given system is varied. For each value of $\frac{\left\langle N_{\text {coll }}\right\rangle}{A}$, the isotropy ratio $R_{E}^{\text {sim }}$ is estimated and compared with the results shown in Fig. 1.

\section{Estimations of the in-medium nucleon-nucleon cross sections, $\sigma_{N N}^{*}$ and the mean free path in the nuclear medium}

The mean number of collisions by nucleons, $\frac{\left\langle N_{\text {coll }}\right\rangle}{A}$, can be related to the effective cross-sections using the "rows on rows" model [26, based on the Glauber assumption [27, 28]:

$$
\frac{\left\langle N_{\text {coll }}\right\rangle}{A}=\frac{1}{2 R_{n}} \cdot \int_{-R_{n}}^{R_{n}} \frac{N_{n}(r) \cdot \sigma_{N N}^{*}}{\sigma_{N N}^{g e o}} \cdot d r
$$

where $R_{n}$ is the radius of the projectile nucleus, $N_{n}$ is the number of nucleons contained in the row at the radial distance $r$ in the nucleus. $\sigma_{N N}^{*}$ is the effective NN collisions cross section to be determined and the geometrical NN collision cross section $\sigma_{N N}^{g e o}=\pi r_{0}^{2}=45 \mathrm{mb}$ with $r_{0}=1.2 \mathrm{fm}$. Assuming a Poisson statistics for the mean 
number of collisions (expected to be small), and performing a Monte-Carlo simulation, a relation between $\frac{\left\langle N_{\text {coll }}\right\rangle}{A}$ and $\sigma_{N N}^{*}$ is obtained. Note that the calculation has been done at a density $\rho=1.2 \rho_{0}$ with $\rho_{0}=0.17 \mathrm{fm}^{-3}$ in order to consider a small compression stage during the reaction. This value corresponds to the mean maximal density coming from transport models at incident around and above $E_{F}(<100 \mathrm{MeV} / \mathrm{A})$ [29]. A smaller value $\left(\rho \approx \rho_{0}\right)$ will only change by a small amount the value of the cross-sections and will not change the conclusions drawn here. It is also worthwhile to mention that the method used here differs from what has been done in 9] where a relation was supposed between the stopping ratio and the mean free path. Here, we check this hypothesis by our complete Monte Carlo implementation and conclude for its validity since the obtained results are very similar, although presenting some differences, especially at low incident energy below $E_{F}$. These discrepancies are indeed observed at low incident energy, in a domain for which our hypothesis concerning the Glauber approximation can be incorrect.

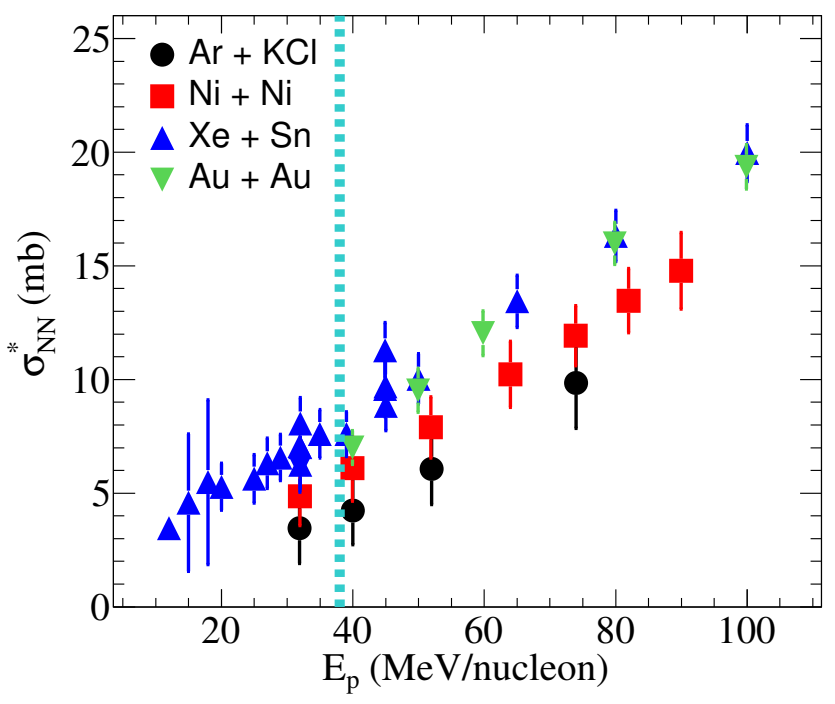

FIG. 2. (Color online) Evolution of the in-medium nucleonnucleon cross section $\sigma_{N N}^{*}$ as a function of the incident projectile energy $E_{P}$. The vertical dotted line corresponds to the Fermi energy.

Fig. 2 shows the evolution of the in-medium NN collisions cross section $\sigma_{N N}^{*}$ as a function of the projectile energy $E_{P}$. An increase of $\sigma_{N N}^{*}$ with the energy of the projectile is obtained. The values, located between 5 and $25 \mathrm{mb}$, are lower than the geometrical cross section $\sigma_{N N}^{g e o}$. A similar mass scaling is observed as already seen in Fig. 1. This is due to the fact that the relation between the mean number of collisions $\frac{\left\langle N_{\text {coll }}\right\rangle}{A}$ and the inmedium NN collisions cross section $\sigma_{N N}^{*}$ is linear due to the Glauber assumption.

The mean free path of the nucleons in the nuclear medium, $\lambda_{N N}^{*}$ is obtained with the following relation:

$$
\lambda_{N N}^{*}=\frac{1}{\rho \sigma_{N N}^{*}}
$$

where $\rho=1.2 \rho_{0}$ is the chosen density of the medium. Fig. 3 displays the evolution of $\lambda_{N N}^{*}$ as a function of the projectile energy. The in-medium mean free path

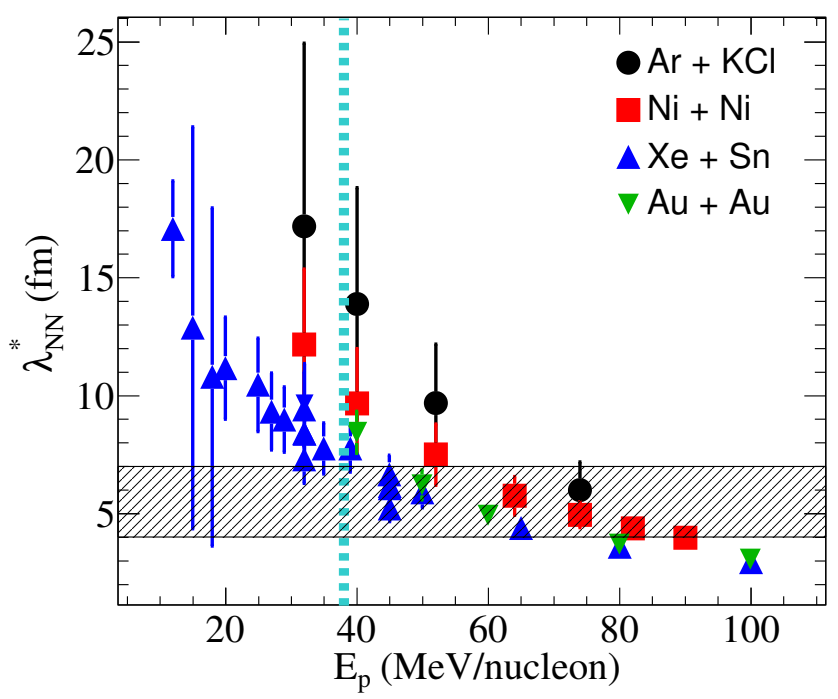

FIG. 3. (Color online) Evolution of the in-medium nucleon mean free path $\lambda_{N N}^{*}$ as a function of the incident projectile energy $E_{P}$. The vertical dotted line corresponds to the Fermi energy. The horizontal hatched zone corresponds to the explored zone in terms of nuclei radius, the lower and upper values correspond to the radius of the $A r$ and $A u$ nuclei, which are respectively the smallest and the biggest nuclei used here.

decreases with the projectile energy. The values are in agreement with known experimental values [9, 30, 31, with theoretical calculations [32] and are here comparable with the radii of the nuclei (between 4 and $7 \mathrm{fm}$ ). This suggests that the energy dissipation of the entrance channel via binary nucleon-nucleon collisions is not complete because there are only few collisions, and, as such that the system cannot be fully thermalized by such processes alone. This could already be anticipated in view of the rather low values of the isotropy ratio in Fig. 1. We can conclude at this point that above the Fermi energy the nuclear medium is partially transparent for the incident nucleons, this effect being due to the Pauli exclusion principle and also possibly to the in-medium effects (density), which are studied in the following section.

\section{Quantification of the in-medium effects}

The results shown in Fig. 2 take into account at the same time the in-medium effect due to the nuclear environment (N-body correlations), but also the Pauli exclusion principle effect (2-body correlations). To separate 
the role of the two effects, we propose the following factorization for the in-medium nucleon-nucleon cross section:

$$
\sigma_{N N}^{*}=\sigma_{N N}^{\text {free }} \times f_{\text {Pauli }} \times \eta
$$

$\sigma_{N N}^{f r e e}$ is the free NN collision cross section (i.e. in vacuum). $f_{\text {Pauli }}$ is the Pauli reduction factor which takes into account the effect of the Pauli exclusion principle on the NN cross section. Finally, $\eta$ is the nuclear reduction factor which takes into account the effect of the nuclear medium (density) on the NN cross section. The estimation of the $f_{\text {Pauli }}$ reduction factor is done by using the semi-classical parametrization of Kikuchi \& Kawai 33. which reads:

$$
f_{\text {Pauli }}= \begin{cases}1-\frac{7}{5} \zeta & , \text { if } \zeta \leq \frac{1}{2} \\ 1-\frac{7}{5} \zeta+\frac{2}{5} \zeta\left(2-\frac{1}{\zeta}\right)^{\frac{5}{2}} & , \text { if } \zeta>\frac{1}{2}\end{cases}
$$

where $\zeta=\frac{E_{F}}{E_{F}+E}$ with $E_{F}$ the Fermi energy and $E$ the kinetic energy of the incident nucleon. This parametrization is based on a geometrical computation of the occupancy of the phase space for the nucleons in the Fermi spheres. Then, the accessible phase space for the scattered nucleons is the space located outside the two Fermi spheres corresponding to the projectile and the target. The factor $f_{\text {Pauli }}$ increases with the projectile energy $\left(f_{\text {Pauli }} \approx 40 \%\right.$ at $E_{P}=50 \mathrm{MeV} /$ nucleon and $f_{\text {Pauli }} \approx 60 \%$ at $E_{P}=100 \mathrm{MeV} /$ nucleon), which means that at low incident energies $(<30 \mathrm{MeV} /$ nucleon $)$, the Pauli exclusion principle forbids most of the NN collisions.

Concerning the free NN cross section, the parametrization of [23, 24] have been used:

$$
\begin{gathered}
\sigma_{p p}\left(E_{p}, \rho=0\right)=\sigma_{n n}\left(E_{p}, \rho=0\right)= \\
{\left[23,5+0,0256\left(18,2-E_{p}^{0,5}\right)^{4}\right] m b} \\
\sigma_{p n}\left(E_{p}, \rho=0\right)=\sigma_{n p}\left(E_{p}, \rho=0\right)= \\
{\left[31,5+0,092 . a b s\left(20,2-E_{p}^{0,53}\right)^{2,9}\right] m b}
\end{gathered}
$$

From $f_{\text {Pauli }}, \sigma_{N N}^{\text {free }}, \sigma_{N N}^{*}$ the $\eta$ nuclear quenching factor can be determined from Eq. (5). Fig. 4 shows the evolution of $\eta$ for all studied systems. Our results have been compared with the parametrization of the in-medium nucleon-nucleon collisions from [34, which reads:

$$
\begin{aligned}
& \sigma_{N N}^{*}=\sigma_{0} \times \tanh \left(\frac{\sigma_{N N}^{\text {free }}}{\sigma_{0}}\right) \\
& \sigma_{0}=y \cdot \rho^{-\frac{2}{3}}
\end{aligned}
$$

Our results are in agreement with the prescription for $\rho=1.2 \rho_{0}$ (as previously for the "rows-on-rows" model)

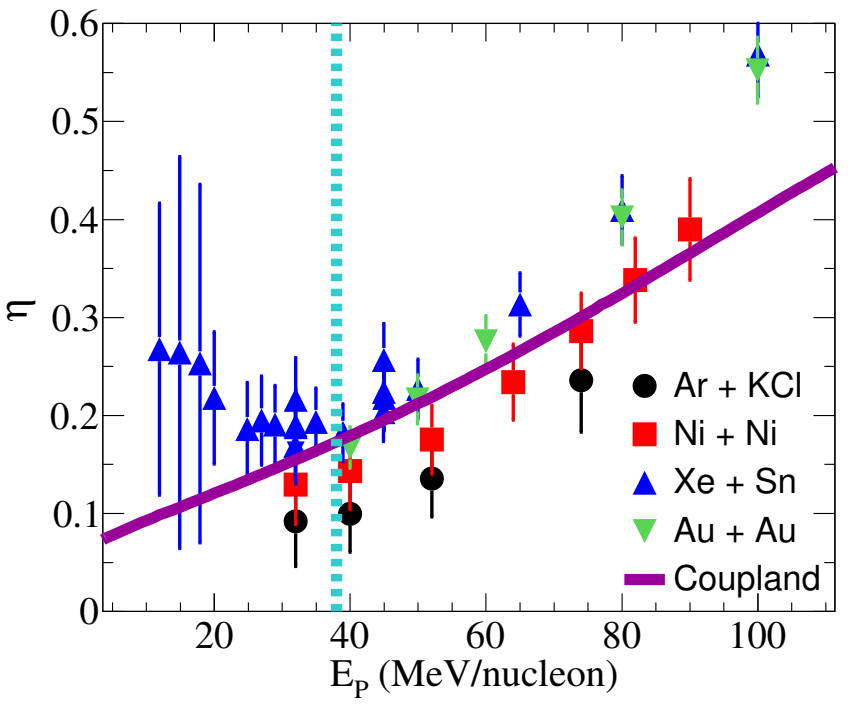

FIG. 4. (Color online) Evolution of the $\eta$ nuclear reduction factor as a function of the incident projectile energy $E_{P}$. The vertical dotted line corresponds to the Fermi energy. The curve corresponds to the comparison of our results with the parametrization of the in-medium nucleon-nucleon cross section of COUPLAND 34 .

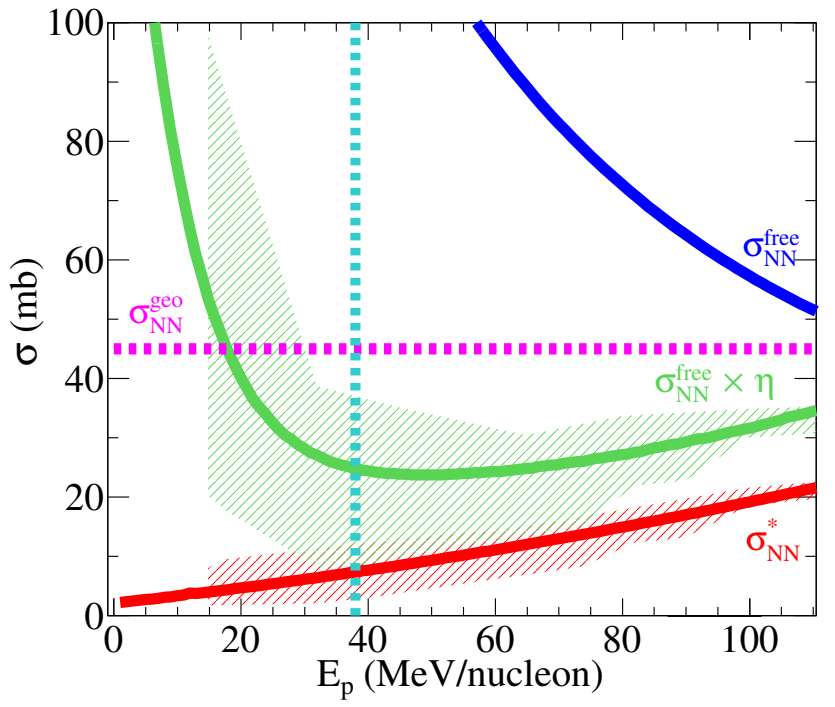

FIG. 5. (Color online) Comparison of the nucleon-nucleon cross sections in the vacuum $\left(\sigma_{N N}^{f r e e}\right)$, corrected by the nuclear reduction factor $\left(\sigma_{N N}^{f r e e} \times \eta\right)$ and in-medium $\left(\sigma_{N N}^{*}\right)$. The hatched zones represent the uncertainties. The horizontal line corresponds to the geometrical nucleon-nucleon cross section $\left(\sigma_{N N}^{g e o}\right)$ while the vertical is the Fermi energy.

and $y=0.9$.

Fig. 5 summarizes the evolution of the NN cross sections in vacuum (blue curve), in the medium taking into account the quenching factor (green curve) and the Pauli exclusion principle (red curve). The filled areas indicate the error bars associated to each cross-section. They 
have been determined by taking the experimental error bars for $\sigma_{N N}^{*}$ from Fig. 2 and propagating them using Eq. (5). Since $f_{\text {Pauli }}$ from Eq. (6) becomes very small at low incident energy, the corresponding error bars on the in-medium $N N$ cross section corrected from Pauli effect (green curve and area) are then very large. The in-medium nucleon-nucleon cross section is dramatically reduced (as observed in [9] and more recently in [10]) as compared to the one in vacuum around the Fermi energy, where the reduction factors $f_{\text {Pauli }}$ and $\eta$ are the more restrictive. As the incident energy increases, the in-medium cross section increases, tending asymptotically at the highest energies considered here ( $\gg 100 \mathrm{MeV} /$ nucleon) towards the free nucleon-nucleon collisions cross section. We therefore confirm the previous results by doing i) a more detailed and complete analysis of the stopping data, ii) a better estimation of all uncertainties and iii) by performing a full Monte Carlo simulation of the NN collisions.

\section{STUDY OF THE IN-MEDIUM PRODUCTION OF LIGHT CLUSTERS}

The production of light clusters during the collision is now investigated. In this analysis, light clusters refers to the hydrogen and helium isotopes: ${ }^{2} \mathrm{H},{ }^{3} \mathrm{H},{ }^{3} \mathrm{He}$ and ${ }^{4} \mathrm{He}$.

In the following, we consider the parallel and perpendicular velocity distributions of the clusters in the center of mass frame with the same central event selection as the previous section. The analysis is performed on 11 systems of the table 1 from $\mathrm{Ni}+\mathrm{Ni}$ up to $\mathrm{Au}+\mathrm{Au}$ and energies from 32 to $90 \mathrm{MeV} /$ nucleon. To this end, a coalescence model for cluster production has been developed and is presented in the next section.

\section{A. A coalescence model for light clusters}

In this section, we present a simple combinatorial coalescence model in momentum space based on the nucleon momentum distributions, obtained from the previous section. The coalescence model takes as a starting point the nucleon-nucleon collisions simulation detailed in the previous section.

\section{Cluster formation and final state interactions}

For each studied system, the total center-of-mass momentum distributions, $f(p)$, of the interacting projectile and target are considered initially as two Fermi spheres (at $T=0)$ separated by the initial relative velocity. Then, $f(p)$ is subsequently modified by in-medium nucleon-nucleon collisions according the results found in the previous section (see section III B). In order to study the kinematics of the light clusters, $2 \leq A_{C} \leq 4$ nucleons are randomly chosen among $f(p)$. These nucleons form a cluster with $Z_{C}$ protons and $A_{C}$ nucleons. Each cluster is characterized by its mean internal kinetic energy defined as:

$$
\langle E\rangle=\sum_{i}^{A_{C}} E_{i}^{c . m} \cdot / A_{C}
$$

where $E_{i}^{c . m}$. is the energy of each nucleon $i$ in the centerof-mass frame of the cluster. From the sum of the momenta of each nucleon in the center-of-mass frame of the total system, we get the velocity of the cluster and its two components $v_{\perp}^{\text {c.m. }}$ and $v_{\|}^{\text {c.m. }}$. In order to be compared in the following with the measured experimental distributions, the simulated distributions are filtered in order to take into account the acceptance of the INDRA array and the detection and identification thresholds.

The coalescence process is assumed to be a fast process and, as such, final state interaction (namely Coulomb interaction) between the cluster and the rest of the system has to be taken into account. We choose to simulate the rest of the system as an expanding uniform charged sphere $\left(Z_{S}, A_{S}\right)$ where $Z_{S}=Z_{P}+Z_{T}-Z_{C}$ (resp. $A_{S}=A_{P}+A_{T}-A_{C}$ ) is the number of charge (resp. nucleons) of this "source". The "source" is in expansion with a velocity $v_{\text {exp }}$ in order to simulate the Coulomb repulsion between all the charged fragments produced in the reaction and also the possible occurrence of a radial flow [35, 36]. In the position space, the source has a given radius $R_{S}$ at $t=0$. The considered cluster is then randomly placed inside the source and the time propagation of the cluster is computed using the initial velocity $v_{C}(0)=\sum_{i=1}^{A_{C}} \frac{p_{i}^{c . m} .}{m}$ with $p_{i}^{c . m}$. the momentum of the nucleon $i$ and $m$ the nucleon mass. The propagation is stopped when the coulomb potential interaction energy between the cluster and the rest of the system is lower than $1 \mathrm{MeV}$.

The model has thus two free parameters: $v_{\text {exp }}$ and $\langle E\rangle$.

\section{Determination of $v_{\exp }$ and $\langle E\rangle$}

To obtain the distribution of these two parameters, a series of calculation is performed, system by system, over a large domain of variation for $v_{\exp }$ and $\langle E\rangle$. Values of $\left(\langle E\rangle, v_{\text {exp }}\right)$ are discretized in bins of $1 \mathrm{MeV}$ and $1 \mathrm{~cm} / \mathrm{ns}$ and sampled with prior uniform distributions. The experimental and simulated (filtered) perpendicular $\left(v_{\perp}^{c . m .}\right)$ and parallel $\left(v_{\|}^{c . m .}\right)$ velocity distributions are compared and a $\chi^{2}$ value is determined.

The comparison is made only on the forward (positive) part of the velocity distributions, in order to avoid thresholds issues in the backward (negative) part of the distributions. A likelihood $\mathcal{L}\left(\langle E\rangle, v_{\text {exp }}\right)=\exp \left(-\frac{\chi^{2}}{2}\right)$ for each couple of values is obtained.

Fig. 6(a) shows for the system ${ }^{129} \mathrm{Xe}+{ }^{119} \mathrm{Sn}$ at $50 \mathrm{MeV} /$ nucleon and for the case of the ${ }^{3} \mathrm{He}$ the likelihood map $\mathcal{L}\left(\langle E\rangle, v_{\text {exp }}\right)$. In this figure are also presented 

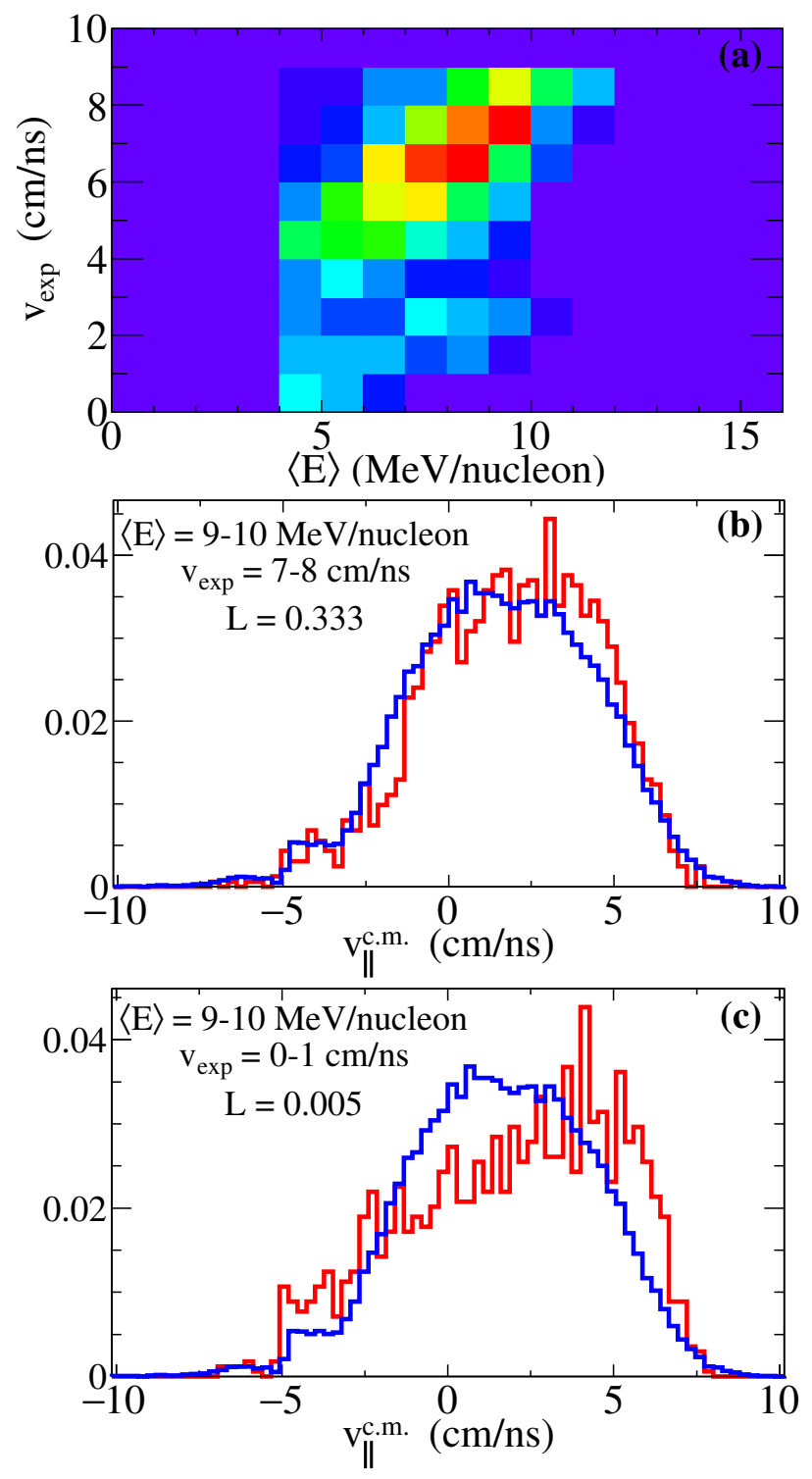

FIG. 6. (Color online) Example with the ${ }^{3} \mathrm{He}$ cluster for the system ${ }^{129} \mathrm{Xe}+{ }^{119} \mathrm{Sn}$ at $50 \mathrm{MeV} /$ nucleon of the procedure followed for the determination of the parameters of the coalescence model. (a) Likelihood map. (b) Superimposition of the normalized experimental INDRA (blue) and simulated (red) parallel velocity distributions for a good likelihood value $(\mathcal{L}=0.333)$. (c) Case of a bad likelihood value $(\mathcal{L}=0.005)$.

the superimposition of the normalized experimental INDRA and simulated distribution for $v_{\|}^{c . m}$. for two given $\left(\langle E\rangle, v_{\text {exp }}\right)$ couples. We can observe for the case of the bad likelihood value (Fig. 6. (c)) that the cluster undergoes a too high repulsion from the source, meaning that the charge seen by the cluster is too important and that the source does not expand fast enough. In the other hand, for the case of the good likelihood value (Fig. 6(b)) that the agreement is better.

In order to not bias the simulation by fixing a unique value for the $\langle E\rangle$ and $v_{\exp }$ parameters, the final distributions of the wanted observables are then built by adding all the distributions associated with a given $\left(\langle E\rangle, v_{\text {exp }}\right)$ and weighted by the associated likelihood values. It is then possible to obtain the distribution of the two $v_{\exp }$ and $\langle E\rangle$ parameters of the coalescence model by projecting the likelihood distribution on each of the two axis. The projection on the $\mathrm{X}$-axis gives the distribution of the mean internal kinetic energy of the nucleons in the cluster $\langle E\rangle$. The projection on the Y-axis gives the distribution of the source expansion velocity $v_{\exp }$.

\section{Results}

Figs. 7, 8,9 and 10 display the results of the coalescence model for all the studied clusters $\left({ }^{2} \mathrm{H},{ }^{3} \mathrm{H},{ }^{3} \mathrm{He},{ }^{4} \mathrm{He}\right)$ for the system ${ }^{129} \mathrm{Xe}+{ }^{119} \mathrm{Sn}$ at $50 \mathrm{MeV} /$ nucleon. The optimization of the parameters by means of the likelihood procedure has been done on the forward part of the two velocity distributions mentioned before, namely $v_{\|}^{c . m}$. and $v_{\perp}^{\text {c.m. }}$.

On one hand, we observe a good agreement between the experimental and the simulated data is when considering the tritons (Fig. 8) and the helium-3 (Fig. 9). On the other hand, the agreement between the model and the experimental data is not satisfactory for the deuterons and the alpha clusters (Fig.77and Fig. 10). Regarding the deuterons, the parallel velocity, the energy and the angular distributions are not well reproduced. For the alpha cluster, even if the energy of the latter is well reproduced by the model, there is a strong disagreement between the coalescence model and the experimental data regarding the parallel velocity and the angular distributions. The fact that these clusters are not well reproduced by the coalescence model is not so surprising since the main production mode of the deuterons and the alpha is not the coalescence but rather evaporation [37, 38.

In the following, we will thus focus on the characteristics of the tritons and the helium- 3 clusters.

\section{B. In-medium $A_{C}=3$ clusters characteristics}

As mentioned before, the distribution of the two parameters $v_{\text {exp }}$ and $\langle E\rangle$ of the model are extracted from the likelihood procedure.

First, we focus on the expansion velocity of the source $v_{\text {exp }}$. Its evolution (the plots are not shown) as a function of the projectile energy and the difference between the systems does not present qualitatively and quantitatively a single trend. However, the uncertainties (standard deviation of the projection of the likelihood map (see Fig. 6(a))) on the expansion velocity axis) associated to each value, lead to a constant non-zero value regarding $v_{\text {exp }}$. Thus, the expansion velocity of the source does not seem to be a decisive quantity in the coalescence model, but it must necessarily be non-zero in order to reproduce 

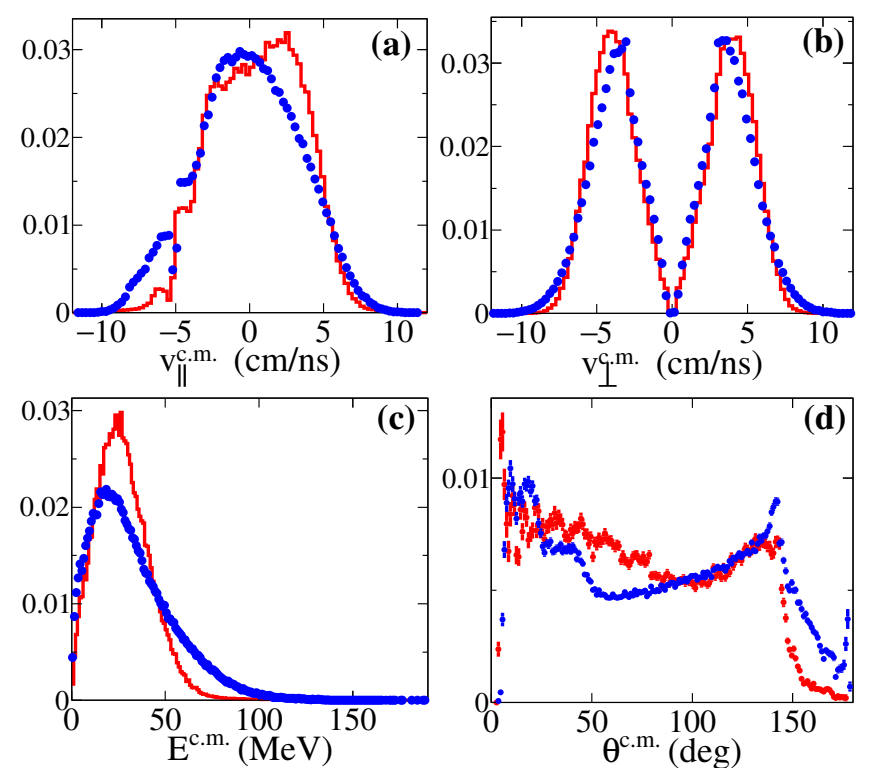

FIG. 7. (Color online) Comparison between the coalescence model and INDRA data for the deuterons in central ${ }^{129} \mathrm{Xe}$ $+{ }^{119} \mathrm{Sn}$ collisions at $50 \mathrm{MeV} /$ nucleon. Blue points are experimental data, red histogram corresponds to the model for the parallel (a) and perpendicular (b) velocities, the kinetic energy (c) and $\theta^{\text {c.m. }}$ angle (d). All quantities are calculated in the center-of-mass frame of the reaction. Y-axes represent normalized yields in arbitrary units.
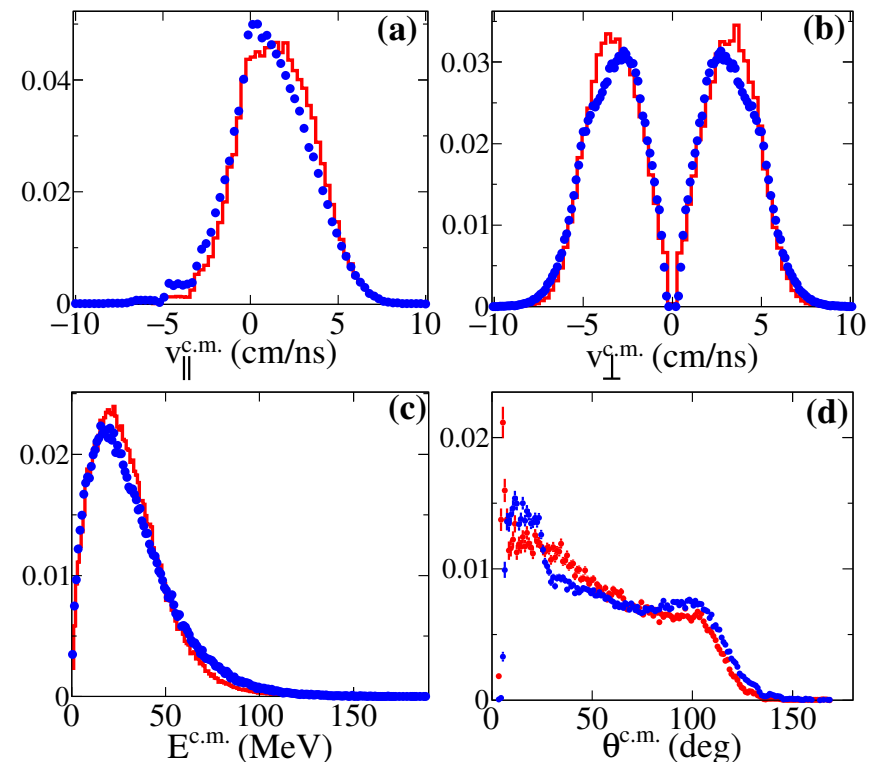

FIG. 8. (Color online) Same as Fig. 7 for the tritons.

the experimental data. We observed that in the case of the $A_{C}=3$ clusters, this velocity evolves between 5 and $8 \mathrm{~cm} / \mathrm{ns}$.

Fig. 11 shows the evolution of the mean internal kinetic energy $\langle E\rangle$. The uncertainties associated to each point correspond here to the standard deviation of the projec-
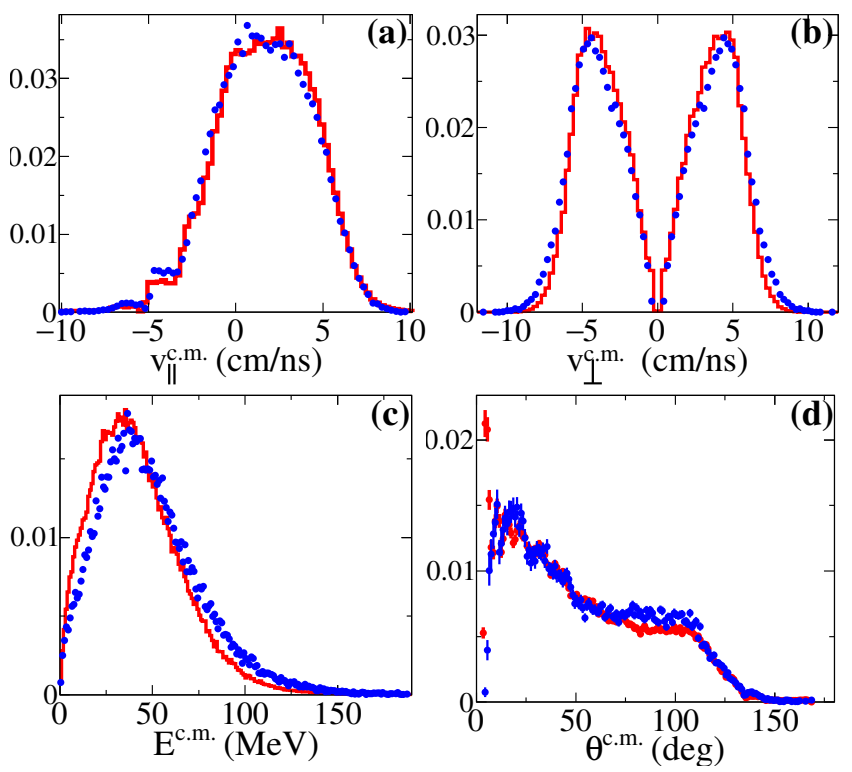

FIG. 9. (Color online) Same as Fig. 7 for the helium-3.
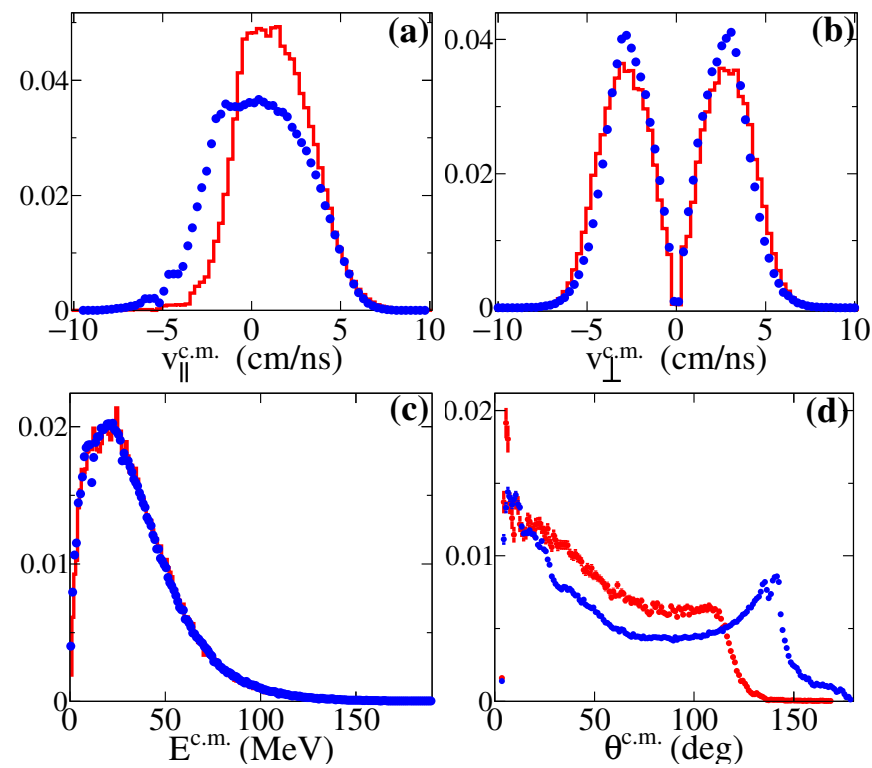

FIG. 10. (Color online) Same as Fig. 7 for the alpha.

tion of the likelihood map (see Fig. 6(a)) on the $\langle E\rangle$ axis. Rather similar values of $\langle E\rangle$ are obtained whatever the system considered for both ${ }^{3} \mathrm{H}$ and ${ }^{3} \mathrm{He}$. Indeed, a coalescence process should not depend either on the size nor on the isospin content of the system. It should also not depend on the isospin of the clusters. Here, a strong evolution is observed as a function of the incident energy. Using a mass dependent momentum distribution based on the harmonic oscillator (HO) prescription [39, one obtains $\langle E\rangle_{H O}^{A=3}=11.1 \mathrm{MeV} /$ nucleon: a larger value than the present results.

Two possible explanations regarding this last observation are proposed hereafter: 

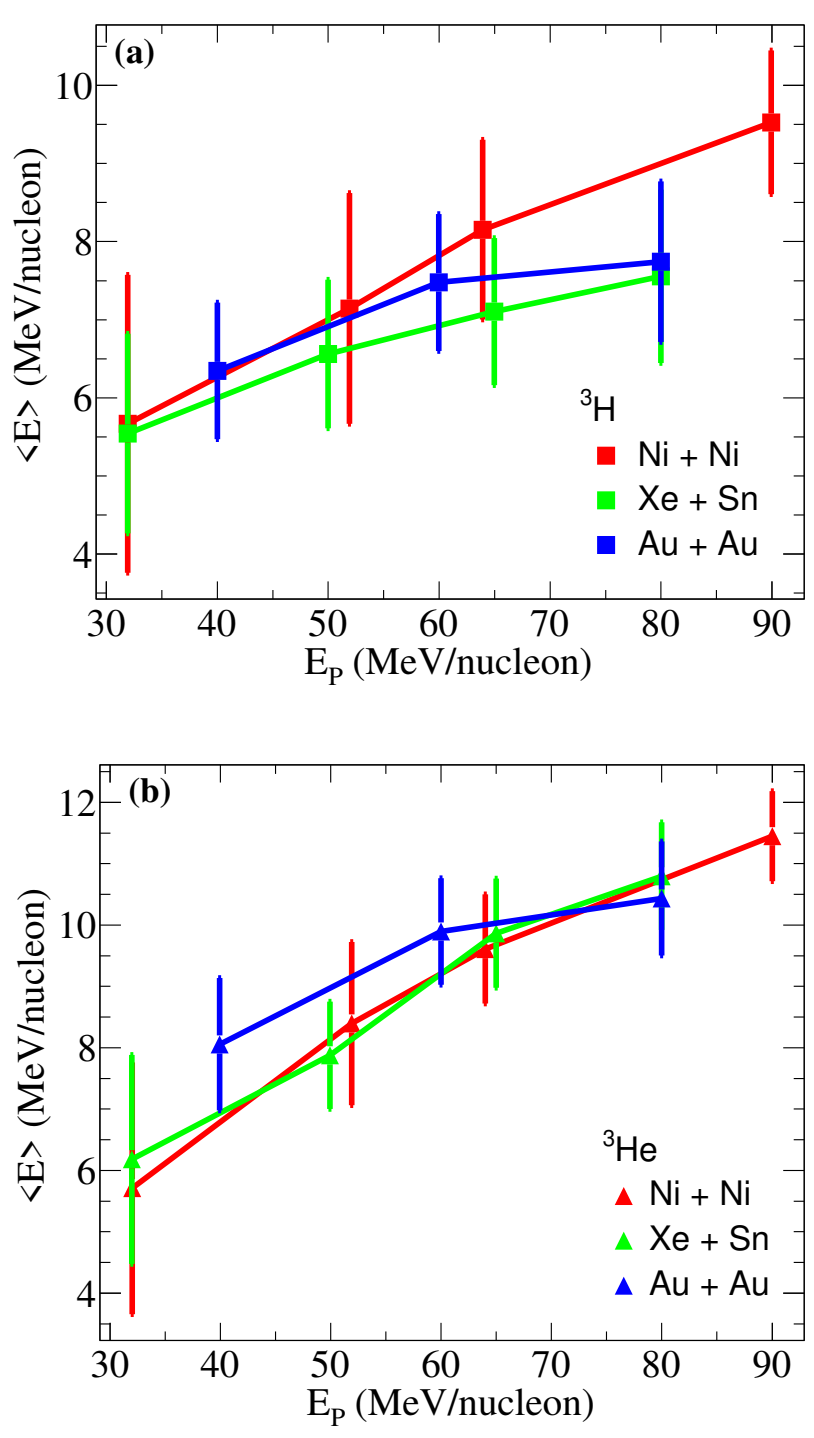

FIG. 11. (Color online) Evolution of the mean internal kinetic energy $\langle E\rangle$ (see text) as a function of projectile energy for three different systems. (a) Case of the ${ }^{3} \mathrm{H}$, (b) Case of the ${ }^{3} \mathrm{He}$.

i) this could be related to an entrance channel effect.

ii) this could be also related to in-medium effect during the cluster production.

The entrance channel effect (case i)) is related to the fact that the relative momentum between the two Fermi spheres (projectile ad target) increases with the bombarding energy. Thus, the mean "distance" in the momentum space between all the nucleons increases also. Nevertheless, the smooth evolution of $\langle E\rangle$ with $E_{P}$ may be explained by the fact that we are considering some in-medium effects in the coalescence model. Indeed, the coalescence process occurs after the nucleon-nucleon collision stage (see section IIIB realized with the results obtained in the previous section (see Fig. 22). The scattered nucleons are thus populating the free space between the two Fermi spheres, reducing the mean "distance" between all the nucleons available for the coalescence process.

The in-medium effects (case ii)) are related to two different points. The first is that, in the sudden approximation and for central collisions (hypothesis of the model), the momentum distributions of the nuclei are not modified. However, as the model is constrained to fit the experimental data (see section. IV A 2), some compression effects in the entrance channel are possible. In this case, the mean distance in the position space decreases with increasing bombarding energy, enhancing the relative momentum between all the nucleons and thus the mean internal kinetic energy $\langle E\rangle$. This explain the trend of the evolution of $\langle E\rangle$ with $E_{P}$. The second point is that, all the values for $\langle E\rangle$ obtained are smaller than the $\mathrm{HO}$ one $\langle E\rangle_{H O}^{A=3}$, the latter being the one expected in vacuum. This suggests that the momentum distribution of the cluster $f_{C}^{*}(p)$ in the nuclear medium differs from those in vacuum $f_{C}(p)$. Similar conclusions have been drawn in [40] where the question of the binding energies of the clusters is raised.

Finally, it would be interesting to study the link between the values $\langle E\rangle$ obtained in this work and the $P_{0}$ momentum of coalescence model used in the litterature 41] whose value is generally of the order of the Fermi momentum. This could constitute the subject of a future study.

It is important to mention here that huge effort are devoted nowadays to quantify the in-medium modification of light clusters binding energies since it has important implications on the EoS [14, 42, 44].

\section{SUMMARY}

In this work, we have studied the in-medium effects in nuclear reactions in the Fermi energy range using the large available INDRA database. The nucleon-nucleon two-body dissipation mode and the cluster production in the nuclear medium were studied in central collisions. These last ones were selected with the help of the total multiplicity of charged particles, in which the amount of energy dissipation is supposedly the most important.

The first part of the analysis was devoted to a study of the in-medium elastic nucleon-nucleon collisions, $\sigma_{N N}^{*}$, using the energy isotropy ratio of the detected protons. It was found that $\sigma_{N N}^{*}$ is significantly reduced as compared to the corresponding vacuum value. The reduction is composed of two terms: the Pauli blocking factor $\left(f_{\text {Pauli }}\right)$ and the in-medium quenching $(\eta)$ factors. Typical values range from very small around the Fermi energy $\left(\sigma_{N N}^{*} \approx 5 \mathrm{mb}\right)$ up to $\approx 20 \mathrm{mb}$ at $100 \mathrm{MeV} /$ nucleon). The deduced nuclear quenching factor $\eta$ was compared rather successfully with existing parametrizations. 
The mean free path in the nuclear medium $\lambda_{N N}^{*}$ was deduced from the values of $\sigma_{N N}^{*}$. It is found to decrease when increasing the projectile energy. However, $\lambda_{N N}^{*}$ is of the same order of magnitude as the typical radius of the nucleus, meaning that the amount of energy dissipation via binary nucleon-nucleon elastic collisions is not large enough above the Fermi energy to ensure full stopping.

In the second part of this work, a coalescence model has been developed to study the kinematical characteristics of the clusters produced in central nuclear reactions. We observed that the $A_{C}=3$ clusters $\left({ }^{3} \mathrm{H}\right.$ and $\left.{ }^{3} \mathrm{He}\right)$ are well reproduced by the coalescence model, unlike deuterons and alpha. This is mainly due to the fact that these latter are mainly produced by evaporation and not by coalescence. We observed that a non-zero expansion velocity of the source simulating the fragments was mandatory to reproduce the data although no precise value was obtained but rather a range of velocity; $5 \leq v_{\text {exp }} \leq 8 \mathrm{~cm} / \mathrm{ns}$.

We found also that the mean internal kinetic energy of the nucleons inside the cluster $\langle E\rangle$ is almost independent of the size of the system. It however increases with bombarding energies and this could point out dynamical and/or density effects. Such a trend should be further investigated in the framework of microscopic transport models in order to bring constraints on the nuclear matter equation of state used in theoretical models describing astrophysical objects where clusters are of great importance (neutrino opacity [45]).
Then, one should mention that the $A_{C}=3$ isobaric mirror clusters ${ }^{3} \mathrm{H}\left(\frac{N}{Z}=2\right)$ and ${ }^{3} \mathrm{He}\left(\frac{N}{Z}=\frac{1}{2}\right)$ may constitute very suitable probes to study the isospin degree of equilibration in central heavy ion collisions. Indeed, these last show an important $\frac{N}{Z}$ difference although a quite similar binding energy $(8.48 \mathrm{MeV}$ for the ${ }^{3} \mathrm{H}$ and $7.72 \mathrm{MeV}$ for the ${ }^{3} \mathrm{He}$ ). As such, the comparison between the two species is expected to be sensitive to the isospin content of the nuclei of the entrance channel. In particular, by combining various isospin content of the projectile and/or the target, it is possible to test the degree of isospin equilibration achieved in central collisions. Given the fact that the developed coalescence model allow to well reproduce the kinematic characteristics of these clusters, it could be interesting to lead such study since the Coulomb effects are easily quantifiable. To this end, the use of the large angular coverage of INDRA, but also new powerful multi-detectors such as FAZIA [46, 47] is certainly a very promising avenue.

\section{ACKNOWLEDGMENTS}

We acknowledge the support from Région Normandie under RIN/FIDNEOS funding. We gratefully acknowledge support from the CNRS/IN2P3 Computing Center (Lyon - France) for providing computing and dataprocessing resources needed for this work.
[1] "Dynamics and Thermodynamics with Nuclear Degrees of Freedom," The European Physical Journal A 30, 1342 (2006)

[2] Jrme Margueron, Rudiney Hoffmann Casali, and Francesca Gulminelli, "Equation of state for dense nucleonic matter from metamodeling. II. Predictions for neutron star properties," Physical Review C 97 (2018), 10.1103/PhysRevC.97.025806

[3] A. Ono, "Dynamics of clusters and fragments in heavyion collisions," Progress in Particle and Nuclear Physics 105, 139-179 (2019)

[4] W. Bauer, "Nuclear Stopping at Intermediate Beam Energies," Physical Review Letters 61 (1988).

[5] S. Kumar, S. Kumar, and R.K. Puri, "Effect of the symmetry energy on nuclear stopping and its relation to the production of light charged fragments," Physical Review C 81 (2010), 10.1103/PhysRevC.81.014601

[6] P. Eudes, Z. Basrak, F. Sbille, V. de la Mota, and G. Royer, "Comprehensive analysis of fusion data well above the barrier," Physical Review C 90 (2014), 10.1103/PhysRevC.90.034609

[7] L. Manduci, O. Lopez, A. Chbihi, M. F. Rivet, R. Bougault, J. D. Frankland, B. Borderie, E. Galichet, M. La Commara, N. Le Neindre, and others (INDRA Collaboration), "Reaction and fusion cross sections for the near-symmetric system Xe $129+$ Sn nat from 8 A to
35 A MeV," Physical Review C 94 (2016), 10.1103/PhysRevC.94.044611

[8] G. Lehaut, D. Durand, O. Lopez, E. Vient, A. Chbihi, J. D. Frankland, E. Bonnet, B. Borderie, R. Bougault, E. Galichet, et al., "Study of nuclear stopping in central collisions at intermediate energies," Physical Review Letters 104 (2010), 10.1103/PhysRevLett.104.232701

[9] O. Lopez, D. Durand, G. Lehaut, B. Borderie, J. D. Frankland, M. F. Rivet, R. Bougault, A. Chbihi, E. Galichet, D. Guinet, and others (INDRA Collaboration), "Inmedium effects for nuclear matter in the fermi-energy domain," Physical Review C 90 (2014), 10.1103/PhysRevC.90.064602

[10] Z. Basrak, P. Eudes, and V. de la Mota, "Aspects of the momentum dependence of the equation of state and of the residual NN cross section, and their effects on nuclear stopping," Physical Review C 93, 054609 (2016).

[11] K. Hagel, R. Wada, L. Qin, J. B. Natowitz, S. Shlomo, A. Bonasera, G. Röpke, S. Typel, Z. Chen, M. Huang, et al., "Experimental determination of in-medium cluster binding energies and mott points in nuclear matter," Phys. Rev. Lett. 108, 062702 (2012)

[12] R. Wada, K. Hagel, L. Qin, J. B. Natowitz, Y. G. Ma, G. Röpke, S. Shlomo, A. Bonasera, S. Typel, Z. Chen, et al., "Nuclear matter symmetry energy at $0.03 \leq$ $\rho / \rho_{0} \leq 0.2, "$ Phys. Rev. C 85, 064618 (2012) 
[13] H. Pais, F. Gulminelli, C. Providência, and G. Röpke, "Light clusters in warm stellar matter: Explicit mass shifts and universal cluster-meson couplings," Phys. Rev. C 97, 045805 (2018)

[14] G. Röpke, "Clusters in nuclear matter and mott points," Physics of Particles and Nuclei 46, 772-776 (2015)

[15] K. Hagel, J. B. Natowitz, and G. Röpke, "The equation of state and symmetry energy of low-density nuclear matter," The European Physical Journal A 50, 39 (2014).

[16] J. Pouthas, B. Borderie, R. Dayras, E. Plagnol, M. F. Rivet, F. Saint-Laurent, J. C. Steckmeyer, G. Auger, Ch O. Bacri, S. Barbey, et al., "INDRA, a $4 \pi$ charged product detection array at GANIL," Nuclear Instruments and Methods in Physics Research Section A: Accelerators, Spectrometers, Detectors and Associated Equipment 357, 418-442 (1995)

[17] J. Pouthas, A. Bertaut, B. Borderie, P. Bourgault, B. Cahan, G. Carles, D. Charlet, D. Cussol, R. Dayras, and M. Engrand, "The electronics of the INDRA $4 \pi$ detection array," Nuclear Instruments and Methods in Physics Research Section A: Accelerators, Spectrometers, Detectors and Associated Equipment 369, 222-247 (1996).

[18] M Prlog, B Borderie, M. F. Rivet, G Tbcaru, A Chbihi, M Elouardi, N Le Neindre, O Lopez, E Plagnol, L TassanGot, and others, "Response of CsI (Tl) scintillators over a large range in energy and atomic number of ions. Part I: recombination and delta electrons," Nuclear Instruments and Methods in Physics Research Section A: Accelerators, Spectrometers, Detectors and Associated Equipment 482, 674-692 (2002)

[19] M. Prlog, B. Borderie, M. F. Rivet, G. Tbcaru, A. Chbihi, M. Elouardi, N. Le Neindre, O. Lopez, E. Plagnol, L. Tassan-Got, and others, "Response of CsI (Tl) scintillators over a large range in energy and atomic number of ions. Part II: calibration and identification in the INDRA array," Nuclear Instruments and Methods in Physics Research Section A: Accelerators, Spectrometers, Detectors and Associated Equipment 482, 693-706 (2002)

[20] O. Lopez, M. Prlog, B. Borderie, M.F. Rivet, G. Lehaut, G. Tabacaru, L. Tassan-got, P. Pawlowski, E. Bonnet, R. Bougault, and others, "Improving isotopic identification with INDRA SiliconCsI( Tl ) telescopes," Nuclear Instruments and Methods in Physics Research Section A: Accelerators, Spectrometers, Detectors and Associated Equipment 884, 140-149 (2018)

[21] B. Borderie and M.F. Rivet, "Nuclear multifragmentation and phase transition for hot nuclei," Progress in Particle and Nuclear Physics 61, 551-601 (2008).

[22] V. Metag, "Nucleus-nucleus collisions in the SIS energy regime," Nuclear Physics A 630, 1-14 (1998).

[23] G. Q. Li and R. Machleidt, "Microscopic calcultion of inmedium proton-proton cross sections," Physical Review C 49, 566-569 (1994).

[24] G. Q. Li and R. Machleidt, "Microscopic calculation of in-medium nucleon-nucleon cross sections," Physical Review C 48, 1702-1712 (1993).

[25] J. Dudouet and D. Durand, "Model for particle production in nuclear reactions at intermediate energies: Application to c-c collisions at $95 \mathrm{mev} /$ nucleon," Phys. Rev. C 94, 014616 (2016)

[26] J. Hufner and J. Knoll, "Rows on rows - A theory for collisions between heavy ions at high energy," Nuclear Physics A 290, 460-492 (1977).
[27] R. J. Glauber and G. Matthiae, "High-energy scattering of protons by nuclei," Nuclear Physics B 21, 135-157 (1970)

[28] I. Brissaud, R. Bimbot, Y. Le Bornec, B. Tatischeff, and N. Willis, "Use of Glauber approximation at low energies," Physical Review C 11, 1537 (1975).

[29] D. Durand, E. Suraud, and B. Tamain, Nuclear Dynamics in the Nucleonic Regime, Series in Fundamental and Applied Nuclear Physics (Taylor \& Francis, 2000).

[30] P. U. Renberg, D. F. Measday, M. Pepin, P. Schwaller, B. Favier, and C. Richard-Serre, "Reaction cross sections for protons in the energy range 220-570 MeV," Nuclear Physics A 183, 81-104 (1972).

[31] A. Nadasen, P. Schwandt, P. P. Singh, W. W. Jacobs, A. D. Bacher, P. T. Debevec, M. D. Kaitchuck, and J. T. Meek, "Elastic scattering of 80-180 MeV protons and the proton-nucleus optical potential," Physical Review C 23, 1023 (1981).

[32] Arnau Rios and Vittorio Soma, "Self-Consistent Greens Function Calculation of the Nucleon Mean Free Path," Physical Review Letters 108 (2012), 10.1103/PhysRevLett.108.012501.

[33] K. Kikuchi and M. Kawai, Nuclear Matter and Nuclear Reactions, North-Holland research monograph (NorthHolland Pub. Co., 1968).

[34] D. D. S. Coupland, W. G. Lynch, M. B. Tsang, P. Danielewicz, and Yingxun Zhang, "Influence of transport variables on isospin transport ratios," Physical Review C 84 (2011), 10.1103/PhysRevC.84.054603

[35] Nathalie Marie, Mouvement collectif et multifragmentation dans les collisions centrales du systme Xe+Sn 50 MeV par nuclons, Ph.D. thesis, Caen Basse-Normandie, GANIL (1995).

[36] Nicolas Le Neindre, Aspects thermodynamiques de la multifragmentation $\mathrm{Xe}+\mathrm{Sn} 50 \mathrm{~A} . \mathrm{MeV}$, Ph.D. thesis, Caen Basse-Normandie, LPC - Caen (1999).

[37] R. J. Charity, "Systematic description of evaporation spectra for light and heavy compound nuclei," Phys. Rev. C 82, 014610 (2010).

[38] R. Bougault, E. Bonnet, B. Borderie, A. Chbihi, D. Dell'Aquila, Q. Fable, L. Francalanza, J. D. Frankland, E. Galichet, D. Gruyer, and others (INDRA Collaboration), "Light charged clusters emitted in 32 $\mathrm{MeV} /$ nucleon Xe $136,124+\mathrm{Sn} 124,112$ reactions: Chemical equilibrium and production of $\mathrm{He} 3$ and He 6," Physical Review C 97 (2018), 10.1103/PhysRevC.97.024612

[39] Ye S. Golubeva, A. S. Iljinov, E. Ya Paryev, and I. A. Pshenichnov, "Subthreshold production on nuclei by protons," Zeitschrift fr Physik A Hadrons and Nuclei 345, 223-226 (1993)

[40] R Bougault, E Bonnet, B Borderie, A Chbihi, J D Frankland, E Galichet, D Gruyer, M Henri, M La Commara, N Le Neindre, et al., "Equilibrium constants of hydrogen and helium isotopes at low nuclear densities," Journal of Physics G: Nuclear and Particle Physics 47, 025103 (2020)

[41] Terry C. Awes, G. Poggi, C. K. Gelbke, B. B. Back, B. G. Glagola, H. Breuer, and V. E. Viola Jr, "Precompound emission of light particles in the reaction $\mathrm{O} 16+\mathrm{U} 238$ at $20 \mathrm{MeV} /$ nucleon," Physical Review C 24, 89 (1981).

[42] Stefan Typel, "Clusters in nuclear matter and the equation of state," Journal of Physics: Conference Series 420, 012078 (2013) 
[43] Helena Pais, Francesca Gulminelli, Constan ça Providência, and Gerd Röpke, "Full distribution of clusters with universal couplings and in-medium effects," Phys. Rev. C 99, 055806 (2019)

[44] Helena Pais, Rémi Bougault, Francesca Gulminelli, Constana Providncia, Eric Bonnet, Bernard Borderie, Abdelouahad Chbihi, John D. Frankland, Emmanuelle Galichet, Diégo Gruyer, et al., arXiv:1911.10849 [nuclth].

[45] E. J. Lentz, A. Mezzacappa, O. E. Bronson Messer, W. R. Hix, and S. W. Bruenn, "Interplay of Neutrino Opacities in Core-Collapse Supernova Simulations," The As- trophysical Journal 760, 94 (2012)

[46] R. Bougault, G. Poggi, S. Barlini, B. Borderie, G. Casini, A. Chbihi, N. Le Neindre, M. Prlog, G. Pasquali, S. Piantelli, et al., "The FAZIA project in Europe: R\&D phase," The European Physical Journal A 50 (2014), 10.1140/epja/i2014-14047-4.

[47] S. Valdre, G. Casini, N. Le Neindre, M. Bini, A. Boiano, B. Borderie, P. Edelbruck, G. Poggi, F. Salomon, G. Tortone, et al., "The fazia setup: A review on the electronics and the mechanical mounting," Nuclear Instruments and Methods in Physics Research Section A: Accelerators, Spectrometers, Detectors and Associated Equipment 930, $27-36$ (2019) 